\title{
Close-coupling $\boldsymbol{R}$-matrix calculations for electron-ion recombination cross sections
}

\author{
Hong Lin Zhang†, Sultana N Nahar and Anil K Pradhan \\ Department of Astronomy, Ohio State University, Columbus, 43210 OH, USA
}

Received 20 November 1998

\begin{abstract}
Close-coupling (CC) calculations of electron-ion recombination cross sections using the $R$-matrix method are presented and benchmarked with available experimental measurements. The electron-ion recombination process, including resonant and non-resonant recombination may be unified as a natural extension of the coupled-channel approximation, as traditionally employed for photoionization and electron-ion scattering. Recombination cross sections can be calculated to the same accuracy by employing similar eigenfunction expansions for the target ion. Detailed results are obtained for electron recombination with $\mathrm{C} v, \mathrm{C}$ VI, $\mathrm{O}$ vIII and Fe xxv. Several sets of theoretical calculations are reported and discussed: non-relativistic CC in $L S$ coupling, relativistic CC in the Breit-Pauli approximation, with radiative attenuation and fine structure, and the relativistic distorted-wave approximation. The theoretical results are in very good agreement with highly accurate experimental measurements at the Heidelberg test storage ring for $\mathrm{C} v, \mathrm{C}$ VI and $\mathrm{O}$ VIII, and the electron-ion beam trap at Livermore for Fe xxv. We discuss the overall effect of radiation damping of all resonances on effective cross sections and rates, important for $\mathrm{H}$ - and $\mathrm{He}$-like ions. In addition to agreement with experimental data, the validity of the $\mathrm{CC}$ calculations is demonstrated by the continuity between the calculated photorecombination, dielectronic recombination and electron impact excitation cross sections. Certain issues related to the works of Badnell et al (1998 J. Phys. B: At. Mol. Opt. Phys. 31 L239) and Robicheaux (1998 J. Phys. B: At. Mol. Opt. Phys. 31 L109) are also addressed.
\end{abstract}

\section{Introduction}

Experimental measurements of electron-ion recombination cross sections using ion storage rings exhibit detailed resonance structures observed at very high resolution of beam energy (Wolf et al 1991, Kilgus et al 1990, 1993). The experiments measure absolute cross sections and therefore provide ideal tests for theoretical methods, as well as the physical effects included in the calculations. There have been several theoretical calculations for dielectronic recombination (DR) with highly charged ions based on the isolated-resonance distorted-wave approximation (e.g. Pindzola et al 1990, 1992, Badnell et al 1990) and the saddle-point variation method (Mannervik et al 1997), that yield good agreement with experimental data and provide useful benchmarks for the ions concerned. Although resonant recombination via DR generally dominates the recombination process for highly charged ions, for most other atomic systems the background recombination, or radiative recombination (RR), is an inseparable and significant contributor to total $\left(\mathrm{e}^{-}+\right.$ion) recombination. The close-coupling approximation in atomic collision theory enables a consideration of both of these processes. In our previous

$\dagger$ Current address: Applied Theoretical and Computational Physics Division, MS F663, Los Alamos National Laboratory, Los Alamos, NM, USA. 
works on a computationally unified treatment of electron-ion recombination in the closecoupling approximation (Nahar and Pradhan 1994 (NP1), 1995 (NP2)), the primary emphasis was on the calculation of total recombination rates including both the RR and DR processes. For practical applications in astrophysical and laboratory plasmas the unified recombination rates have the further advantage that a single set of recombination rates is tabulated at all temperatures. In recent works these total recombination rates have been computed for all carbon, nitrogen, and oxygen ions (Nahar and Pradhan 1997, Nahar 1999), and for some ions of silicon, sulfur and iron (Nahar 1995, 1996a, b, 1997, Nahar et al 1997, 1998, Nahar and Bautista 1999).

In light of the new experimental studies, however, and given that the unified method for electron-ion recombination is quite general, it is desirable to extend the calculations to elicit detailed features for direct comparison with the measured cross sections. The primary goal of the present work is to thereby demonstrate the accuracy of the method, on a par with the close-coupling (CC) treatment of photoionization and electron impact excitation, as well as to study theoretical issues such as relativistic effects, the distinction between close-coupling and independent resonance treatments, the magnitude of the resonant and the non-resonant (background) cross sections, relatively sparse near-threshold resonance structures as opposed to the dense resonances below the Rydberg series limits, radiation damping of low-lying autoionizing resonances, etc. We present detailed cross sections for carbon and oxygen ions, $\mathrm{Cv}, \mathrm{C}$ vI, $\mathrm{O}$ vIII, for which experimental measurements have been made at the test storage ring (TSR) at Heidelberg (Wolf et al 1991, Kilgus et al 1990, 1993). In a previous work (Zhang and Pradhan 1997) we made similar comparisons for the photorecombination spectra of Ar XIV measured by the storage ring CRYRING at Stockholm (DeWitt et al 1995, 1996) with very good agreement between theory and the absolute measured cross sections. In a letter on radiation damping of autoionizing resonances (Pradhan and Zhang 1997), preliminary results for He-like $\mathrm{C} v$ and $\mathrm{Fe} x \mathrm{xv}$ for individual resonance complexes were presented and compared with the experimental work on the synchrotron storage ring by Mannervik et al (1997) for $\mathrm{C} \mathrm{v}$, and with the measurements on the electron-beam ion trap by Beiersdorfer et al (1992) for Fe xxv; additional results for both ions are presented herein.

Departures from pure $L S$ coupling due to relativistic effects, and resultant fine structure, are of importance for highly charged ions. In the previous work on Ar XIV and Fe xxv (Zhang and Pradhan 1997) the calculations were carried out with the Breit-Pauli (BP) $R$-matrix method (Hummer et al 1993), since these ions are highly charged and the fine structure effects are significant (Zhang 1998). However, the precise onset and magnitude of these effects as a function of $Z$ (nuclear charge) and $z$ (ion charge) require careful study. The present work reports both $L S$-coupling and intermediate-coupling $R$-matrix calculations, the latter in the $\mathrm{BP}$ approximation. For further comparison, fully relativistic calculations are also carried out in $j j$-coupling using a Dirac-Fock-Slater distorted-wave method (Zhang et al 1989, Sampson and Zhang 1995). As the distorted-wave (DW) approach to dielectronic recombination is an independent resonance approximation, in that the autoionization and radiative transition rates are computed independently, we also compare the cross sections derived from these DW DR rates and with those obtained using the $a b$ initio, coupled-channel approximations for $\mathrm{O}$ VIII considered herein.

As shown earlier (Pradhan and Zhang 1997), radiation damping effects are significant for the $\mathrm{H}$ - and He-like ions owing to the highly energetic $n=2 \rightarrow 1$ core transition with a large radiative probability $\Gamma_{\mathrm{r}} \sim 10^{12}-10^{14} \mathrm{~s}^{-1}$ that compete with typical autoionization probabilities of $\Gamma_{\mathrm{a}} \sim 10^{13}-10^{14}$. However, it was shown that the effect is much smaller for low- $Z$ ions such as $\mathrm{C} v$ than for the high- $Z$ ions such as Fe xxv.

In this study of $\mathrm{H}$ - and He-like ions we further investigate the radiation damping effects 
(Pradhan and Zhang 1997). It is pointed out that the recent work by Badnell et al (1998) on the damping of isolated resonance structures is incomplete since several other factors are not considered and no complete calculations are presented for effective recombination cross sections or rates. We also address certain issues related to the use of the Bell and Seaton (BS) theory of DR, and demonstrate its validity, utility and precision. It is emphasized that the recent work of Robicheaux (1998) should not have any practical bearing on DR calculations.

\section{Theory}

The extension of the close-coupling method to electron-ion recombination is described in earlier works (NP1, NP2), together with the details of the unified treatment. Here we present a brief description of the theory relevant to the calculations of electron recombination cross sections of $\mathrm{Cv}, \mathrm{C}$ vi and $\mathrm{O}$ vIII. The calculations are carried out in the close-coupling approximation employing the $R$-matrix method, in $L S$ coupling and in intermediate coupling with the BP Hamiltonian. The target ion is represented by an $N$-electron system, and the total wavefunction expansion, $\Psi(E)$, of the $(N+1)$ electron-ion system of symmetry $S L \pi$ or $J \pi$ may be represented in terms of the target eigenfunctions as

$$
\Psi(E)=A \sum_{i} \chi_{i} \theta_{i}+\sum_{j} c_{j} \Phi_{j}
$$

where $\chi_{i}$ is the target wavefunction in a specific state $S_{i} L_{i} \pi_{i}$ or $J_{i} \pi_{i}$ and $\theta_{i}$ is the wavefunction for the $(N+1)$ th electron in a channel labelled as $S_{i} L_{i}\left(J_{i}\right) \pi_{i} k_{i}^{2} \ell_{i}(S L \pi$ or $J \pi) ; k_{i}^{2}$ being its incident kinetic energy. $\Phi_{j}$ are the correlation functions of the $(N+1)$-electron system that account for short-range correlation and the orthogonality between the continuum and the bound orbitals. Most of the present calculations are in the BP approximation (Pradhan and Zhang 1997, Zhang 1998).

Recombination of an incoming electron to the target ion may occur through non-resonant, background continuum, usually referred to as radiative recombination,

$$
\mathrm{e}^{-}+\mathrm{X}^{2+} \rightarrow h v+\mathrm{X}^{+}
$$

which is the inverse process of direct photoionization, or through the two-step recombination process via autoionizing resonances, i.e. dielectronic recombination:

$$
\mathrm{e}^{-}+\mathrm{X}^{2+} \rightarrow\left(\mathrm{X}^{+}\right)^{* *} \rightarrow\left\{\begin{array}{l}
\mathrm{e}^{-}+\mathrm{X}^{2+} \\
h v+\mathrm{X}^{+}
\end{array}\right.
$$

where the incident electron is in a quasi-bound doubly excited state which leads either to (a) autoionization, a radiationless transition to a lower state of the ion and the free electron, or to (b) radiative stabilization predominantly via decay of the ion core, usually to the ground state, and the bound electron.

In the unified treatment the photoionization cross sections, $\sigma_{\mathrm{PI}}$, of a large number of low- $n$ bound states-all possible states with $n \leqslant n_{\max } \sim 10$-are obtained in the CC approximation as in the Opacity Project (Seaton 1987). Coupled-channel calculations for $\sigma_{\mathrm{PI}}$ include both the background and the resonance structures (due to the doubly excited autoionizing states) in the cross sections. The recombination cross section, $\sigma_{\mathrm{RC}}$, is related to $\sigma_{\mathrm{PI}}$, through detailed balance (Milne relation) as

$$
\sigma_{\mathrm{RC}}(\epsilon)=\frac{\alpha^{2}}{4} \frac{g_{i}}{g_{j}} \frac{(\epsilon+I)^{2}}{\epsilon} \sigma_{\mathrm{PI}}
$$

in Rydberg units; $\alpha$ is the fine structure constant, $\epsilon$ is the photoelectron energy and $I$ is the ionization potential. In the present work, it is assumed that the recombining ion is in 
the ground state, and recombination can take place into the ground or any of the excited recombined $\left(\mathrm{e}^{-}+\right.$ion $)$states. The contributions of these bound states to the total $\sigma_{\mathrm{RC}}$ are obtained by summing over the contributions from individual cross sections. $\sigma_{\mathrm{RC}}$ thus obtained from $\sigma_{\mathrm{PI}}$, including the autoionizing resonances, corresponds to the total (DR $\left.+\mathrm{RR}\right)$ unified recombination cross section.

Recombination into the high- $n$ states must also be included, i.e. $n_{\max }<n \leqslant \infty$ (figure 1 of NP1). To each excited threshold $S_{i} L_{i}\left(J_{i}\right) \pi_{i}$ of the $N$-electron target ion, there corresponds an infinite series of $(N+1)$-electron states, $S_{i} L_{i}\left(J_{i}\right) \pi_{i} \nu \ell$, to which recombination can occur, where $v$ is the effective quantum number. For these states DR dominates the recombination process and the background recombination is negligibly small. The contributions from these states are added by calculating the collision strengths, $\Omega_{\mathrm{DR}}$, employing the precise theory of radiation damping by Bell and Seaton (BS 1985, NP1). Several aspects related to the application of the theory to the calculation of DR collision strengths are described in the references cited. We sketch below a few working expressions derived from the theory.

Including radiative interactions in an ab initio manner in the interaction Hamiltonian for the $\mathrm{e}^{-}+$ion system, a generalized electron-photon scattering matrix $\mathcal{S}$ may be obtained as (Davies and Seaton 1969)

$$
\mathcal{S}=\left(\begin{array}{cc}
\mathcal{S}_{\mathrm{ee}} & \mathcal{S}_{\mathrm{ep}} \\
\mathcal{S}_{\mathrm{pe}} & \mathcal{S}_{\mathrm{pp}}
\end{array}\right)
$$

where $\mathcal{S}_{\mathrm{ee}}$ is the matrix for electron scattering including radiation damping; $\mathcal{S}_{\mathrm{pe}}$ is the matrix for electron capture followed by radiative decay with the emission of a photon; $\mathcal{S}_{\text {ep }}$ is for the inverse process of photoionization and $\mathcal{S}_{\mathrm{pp}}$ for photon-photon scattering. In the absence of interaction with the radiation field $\mathcal{S}_{\text {ee }}$ is the usual scattering matrix $S$. The unitarity condition for $\mathcal{S}$ reflects the conservation of both the incident electron and the emitted photon flux (Davies and Seaton 1969), i.e.

$$
\mathcal{S}_{\mathrm{ee}}^{\dagger} \mathcal{S}_{\mathrm{ee}}+\mathcal{S}_{\mathrm{pe}}^{\dagger} \mathcal{S}_{\mathrm{pe}}=1
$$

The electron-electron scattering matrix, $\mathcal{S}_{\text {ee }}$, may again be partitioned into sub-matrices of open and closed channels, in the energy region below threshold, in terms of its analytic continuation given by the matrix $\chi$ as $\chi_{\mathrm{oo}}, \chi_{\mathrm{oc}}, \chi_{\mathrm{co}}$ and $\chi_{\mathrm{cc}}$, where 'o' denotes the open and ' $c$ ' the closed channels. The open channels are those that are accessible to the incident electron for excitation of a target state in that channel; a closed channel refers to electron energies below an inaccessible target threshold. A given Rydberg series of resonances, converging on to a target threshold $S_{t} L_{t}$, corresponds to the closed channel $\left(S_{t} L_{t}\right) \epsilon \ell$, where $\epsilon=-1 / v^{2}, v$ is the effective quantum number associated with the resonance series. The scattering matrix, $\mathcal{S}_{\mathrm{ee}}$, is then obtained as (BS 1985)

$$
\mathcal{S}_{\mathrm{ee}}=\chi_{\mathrm{oo}}-\chi_{\mathrm{oc}}\left[\chi_{\mathrm{cc}}-g(\nu) \exp (-2 \mathrm{i} \pi \nu)\right]^{-1} \chi_{\mathrm{co}}
$$

where $g(v)=\exp \left(\pi v^{3} \Gamma_{\mathrm{r}} / z^{2}\right) ; \Gamma_{\mathrm{r}}$ is the sum of all possible radiative decay probabilities for the resonance series. These decay probabilities correspond to radiative transitions within the ion core. The outer electron is treated as a 'spectator', in a high- $n$ resonance state, interacting only weakly with the core.

The electron flux trapped in the closed channel resonances may decay radiatively to bound states of the $\mathrm{e}^{-}+$ion system. In multi-channel quantum defect theory we diagonalize the $\chi$ matrix as

$$
\chi_{\mathrm{cc}} N=N \chi_{\mathrm{cc}}
$$

where $\chi_{\mathrm{cc}}$ is a diagonal matrix and $\boldsymbol{N}$ is the diagonalizing matrix with $\boldsymbol{N}^{\mathrm{T}} \boldsymbol{N}=\mathbf{1}$. In terms of $\boldsymbol{N}$ we write $\chi_{\mathrm{oc}}^{\prime}=\chi_{\mathrm{oc}} \boldsymbol{N}$ and $\chi_{\mathrm{co}}^{\prime}=\boldsymbol{N}^{\mathrm{T}} \chi_{\mathrm{co}}$, where $\boldsymbol{N}^{\mathrm{T}}$ is the transpose of $\boldsymbol{N}$. The 


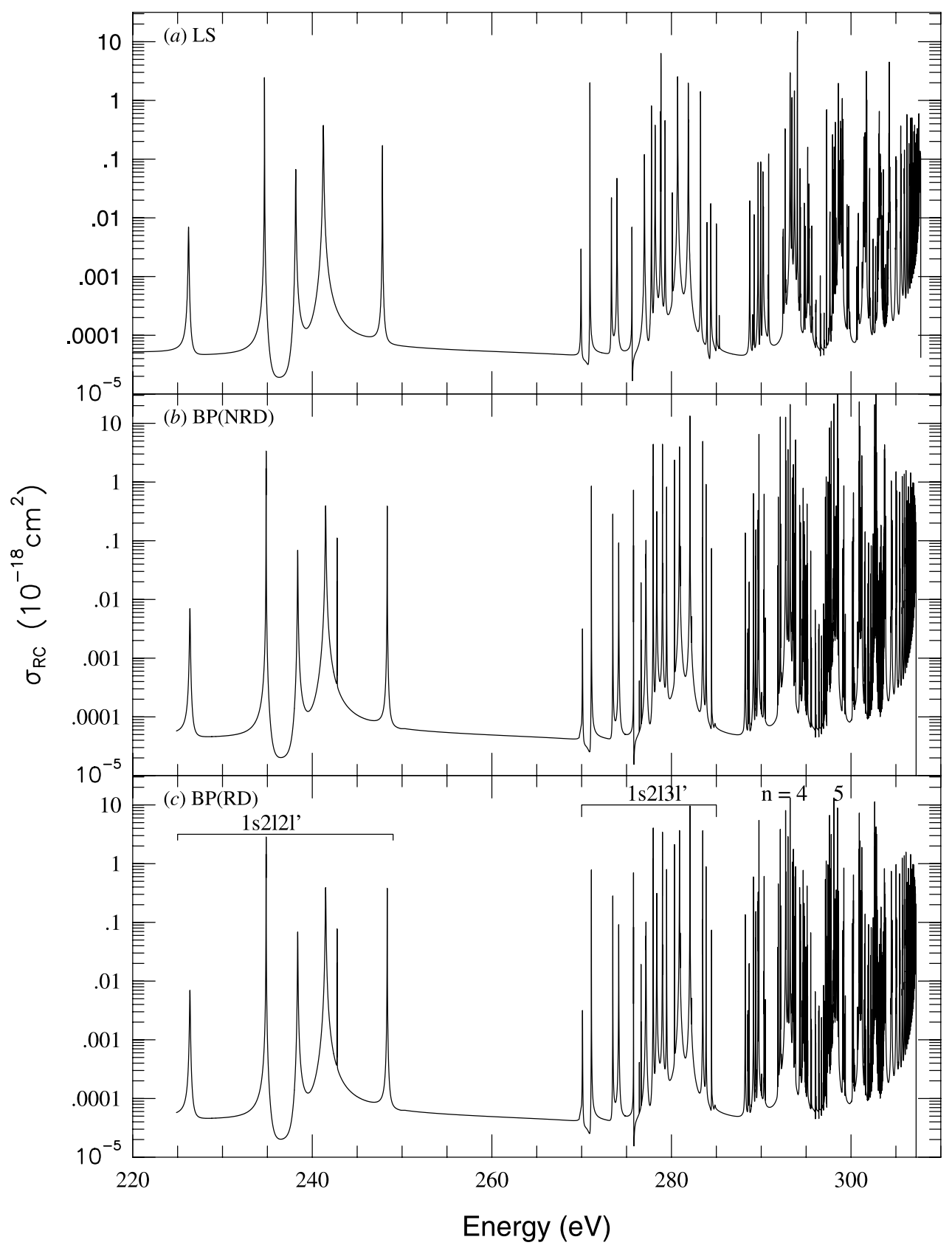

Figure 1. Detailed recombination cross sections, $\sigma_{\mathrm{RC}}$, for $\mathrm{e}^{-}+\mathrm{Cv} \rightarrow \mathrm{C}_{\text {IV }}$ : $(a)$ in non-relativistic $L S$-coupling approximation and in the relativistic Breit-Pauli approximation; $(b)$ without radiation damping, $\mathrm{BP}(\mathrm{NRD})$ and $(c)$ with radiation damping, $\mathrm{BP}(\mathrm{RD})$.

DR probability, for an entrance or incident open channel $\alpha$, is obtained from the unitarity condition as

$$
P_{\alpha}(\mathrm{DR})=\left(\mathbf{1}-\mathcal{S}_{\mathrm{ee}}^{\dagger} \mathcal{S}_{\mathrm{ee}}\right)_{\alpha \alpha} .
$$


Substituting the proper expressions, the DR probability can be written as (NP1, Nahar 1996b)

$$
\begin{aligned}
& P_{\alpha}=G(v) \sum_{\gamma}\left\{\left(\sum_{\gamma^{\prime}} \chi_{\alpha \gamma^{\prime}}^{\prime} \boldsymbol{N}_{\gamma \gamma^{\prime}}\right)\left[\frac{1}{\chi_{\gamma \gamma}-g(\nu) \exp (-2 \pi \mathrm{i} \nu)}\right]\right. \\
& \left.\times\left[\frac{1}{\chi_{\gamma \gamma}^{*}-g(\nu) \exp (+2 \pi \mathrm{i} v)}\right]\left(\sum_{\gamma^{\prime}} \chi_{\gamma^{\prime} \alpha}^{\prime *} \boldsymbol{N}_{\gamma \gamma^{\prime}}^{*}\right)\right\}
\end{aligned}
$$

where $G(v)=g(v)^{2}-1=\exp \left(2 \pi v^{3} \Gamma_{\mathrm{r}} / z^{2}\right)-1$. The summations go over the closed channels $\gamma \gamma^{\prime}$ contributing to DR. The sum over the diagonal elements of all open channels linked to the ground state of the target ion gives the probability of DR through radiative transitions between the excited states and the ground state. As we are interested only in the detailed DR collision strengths, expressions derived for resonance-averaged DR collision strengths, that are useful in the calculation of recombination rate coefficients, are not given here but may be found in (NP1).

The $\Omega_{\mathrm{DR}}$ are calculated, in a self-consistent manner, using the same $\mathrm{CC}$ wavefunction expansion that is used for the calculation of $\sigma_{\mathrm{PI}}$. The DR cross section, in megabarns (Mb), is related to the collision strength, $\Omega_{\mathrm{DR}}$, as

$$
\sigma_{\mathrm{DR}}(i \rightarrow j)(\mathrm{Mb})=\pi \Omega_{\mathrm{DR}}(i, j) /\left(g_{i} k_{i}^{2}\right)\left(a_{0}^{2} / 1 \times 10^{-18}\right)
$$

where $k_{i}^{2}$ is the incident electron energy in Ryd.

Radiation damping of resonances with $n_{\max }<n \leqslant \infty$ is considered using the BS theory as described above. However, for H- and He-like systems the low- $n$ resonances with $n \leqslant n_{\max } \sim 10$ may also be radiatively attenuated depending on $Z$ and $n$ (Pradhan and Zhang 1997). We adopt an approach described by Sakimoto et al (1990) which entails fitting the dipole matrix element to the form

$$
D(E)=D^{0}(E)+\frac{A}{E-Z^{*}} \quad Z=E_{0}-\frac{\mathrm{i}}{2} \Gamma_{\mathrm{a}}
$$

where $E_{0}$ is the resonance position, $D^{0}(E)$ is the background term, $A$ is the residue of the complex pole and $\Gamma_{\mathrm{a}}$ is the autoionization width in Ryd. The radiative decay width $\Gamma_{\mathrm{r}}$ is then obtained by

$$
\Gamma_{\mathrm{r}}=\frac{4 \pi^{2}|A|^{2}}{\Gamma_{\mathrm{a}}}
$$

The second-order radiative effects can then be included by considering

$$
D(E) \longrightarrow \frac{D(E)}{1+L(E)}
$$

where, according to the BS theory (1985), the operator $L(E)$ is given by

$$
L(E)=\pi^{2}\left|D^{0}(E)\right|^{2}+2 \pi^{2} \frac{A^{*} D^{0}(E)}{E-Z}+2 \pi^{2} \frac{|A|^{2}}{(E-Z)\left(Z-Z^{*}\right)} .
$$

The $\Gamma_{\mathrm{r}}$ are obtained in intermediate coupling. A version of the BP $R$-matrix codes developed by Eissner (1998, Pradhan and Zhang 1997) is employed in the present work.

\section{Computations}

The electron-ion recombination calculations are extensions of $\mathrm{CC}$ calculations for photoionization and electron impact excitation. The same eigenfunction expansion for the target (core) ion is employed for all calculations; this makes the computations inherently 
Table 1. Target terms for the $\mathrm{CC}$ eigenfunction expansions of the ions for both non-relativistic $L S$-coupling and relativistic Breit-Pauli (BP) calculations. The target energies are in $\mathrm{eV}$.

\begin{tabular}{|c|c|c|c|c|c|c|c|c|}
\hline \multicolumn{3}{|c|}{ Civ } & \multicolumn{3}{|c|}{$\mathrm{Cv}$} & \multicolumn{3}{|c|}{$\mathrm{O}_{\text {VII }}$} \\
\hline \multirow{2}{*}{$\begin{array}{l}L S \\
1 \mathrm{~s}^{2}\left({ }^{1} \mathrm{~S}\right)\end{array}$} & \multicolumn{2}{|c|}{$\mathrm{BP}$} & \multirow{2}{*}{$\begin{array}{l}L S \\
1 \mathrm{~s}\left({ }^{2} \mathrm{~S}\right)\end{array}$} & \multicolumn{2}{|c|}{$\mathrm{BP}$} & \multirow{2}{*}{$\begin{array}{l}L S \\
1 \mathrm{~s}\left({ }^{2} \mathrm{~S}\right)\end{array}$} & \multicolumn{2}{|c|}{$\mathrm{BP}$} \\
\hline & $1 \mathrm{~s}^{2}\left({ }^{1} \mathrm{~S}_{0}\right)$ & 0.00 & & $1 \mathrm{~s}\left({ }^{2} \mathrm{~S}_{1 / 2}\right)$ & 0.00 & & $1 \mathrm{~s}\left({ }^{2} \mathrm{~S}_{1 / 2}\right)$ & 0.00 \\
\hline $1 \mathrm{~s} 2 \mathrm{~s}\left({ }^{3} \mathrm{~S}\right)$ & $1 \mathrm{~s} 2 \mathrm{~s}\left({ }^{3} \mathrm{~S}_{1}\right)$ & 298.73 & $2 \mathrm{~s}\left({ }^{2} \mathrm{~S}\right)$ & $2 \mathrm{~s}\left({ }^{2} \mathrm{~S}_{1 / 2}\right)$ & 367.36 & $2 \mathrm{~s}\left({ }^{2} \mathrm{~S}\right)$ & $2 \mathrm{~s}\left({ }^{2} \mathrm{~S}_{1 / 2}\right)$ & 653.57 \\
\hline $1 \mathrm{~s} 2 \mathrm{p}\left({ }^{3} \mathrm{P}^{\mathrm{o}}\right)$ & $1 \mathrm{~s} 2 \mathrm{~s}\left({ }^{1} \mathrm{~S}_{0}\right)$ & 304.38 & $2 \mathrm{p}\left({ }^{2} \mathrm{P}^{\mathrm{o}}\right)$ & $2 \mathrm{p}\left({ }^{2} \mathrm{P}_{1 / 2}^{\mathrm{o}}\right)$ & 367.36 & $2 \mathrm{p}\left({ }^{2} \mathrm{P}^{\mathrm{o}}\right)$ & $2 \mathrm{p}\left({ }^{2} \mathrm{P}_{1 / 2}^{\mathrm{o}}\right)$ & 653.57 \\
\hline $1 \mathrm{~s} 2 \mathrm{~s}\left({ }^{1} \mathrm{~S}\right)$ & $1 \mathrm{~s} 2 \mathrm{p}\left({ }^{3} \mathrm{P}_{0}^{\mathrm{o}}\right)$ & 304.39 & $3 \mathrm{~s}\left({ }^{2} \mathrm{~S}\right)$ & $2 \mathrm{p}\left({ }^{2} \mathrm{P}_{3 / 2}^{\mathrm{o}}\right)$ & 367.42 & $3 s\left({ }^{2} S\right)$ & $2 \mathrm{p}\left({ }^{2} \mathrm{P}_{3 / 2}^{\mathrm{o}}\right)$ & 653.73 \\
\hline $1 \mathrm{~s} 2 \mathrm{p}\left({ }^{1} \mathrm{P}^{\mathrm{o}}\right)$ & $1 \mathrm{~s} 2 \mathrm{p}\left({ }^{3} \mathrm{P}_{1}^{\mathrm{o}}\right)$ & 304.39 & $3 p\left({ }^{2} \mathrm{P}^{\mathrm{o}}\right)$ & $3 \mathrm{~s}\left({ }^{2} \mathrm{~S}_{1 / 2}\right)$ & 435.41 & $3 p\left({ }^{2} \mathrm{P}^{\mathrm{o}}\right)$ & $3 \mathrm{~s}\left({ }^{2} \mathrm{~S}_{1 / 2}\right)$ & 774.65 \\
\hline $1 \mathrm{~s} 3 \mathrm{~s}\left({ }^{3} \mathrm{~S}\right)$ & $1 \mathrm{~s} 2 \mathrm{p}\left({ }^{3} \mathrm{P}_{2}^{\mathrm{o}}\right)$ & 304.41 & $3 \mathrm{~d}\left({ }^{2} \mathrm{D}\right)$ & $3 \mathrm{p}\left({ }^{2} \mathrm{P}_{1 / 2}^{\mathrm{o}}\right)$ & 435.41 & $3 \mathrm{~d}\left({ }^{2} \mathrm{D}\right)$ & $3 p\left({ }^{2} P_{1 / 2}^{o}\right)$ & 774.65 \\
\hline $1 \mathrm{~s} 3 \mathrm{~s}\left({ }^{1} \mathrm{~S}\right)$ & $1 \mathrm{~s} 2 \mathrm{p}\left({ }^{1} \mathrm{P}_{1}^{\mathrm{o}}\right)$ & 307.90 & $4 s\left({ }^{2} S\right)$ & $3 \mathrm{p}\left({ }^{2} \mathrm{P}_{3 / 2}^{\mathrm{o}}\right)$ & 435.43 & $4 s\left({ }^{2} S\right)$ & $3 \mathrm{p}\left({ }^{2} \mathrm{P}_{3 / 2}^{\mathrm{o}}\right)$ & 774.71 \\
\hline $1 \mathrm{~s} 3 \mathrm{p}\left({ }^{3} \mathrm{P}^{\mathrm{o}}\right)$ & $1 \mathrm{~s} 3 \mathrm{~s}\left({ }^{3} \mathrm{~S}_{1}\right)$ & 352.05 & $4 p\left({ }^{2} P^{o}\right)$ & $3 \mathrm{~d}\left({ }^{2} \mathrm{D}_{3 / 2}\right)$ & 435.43 & $4 p\left({ }^{2} P^{o}\right)$ & $3 \mathrm{~d}\left({ }^{2} \mathrm{D}_{3 / 2}\right)$ & 774.71 \\
\hline $1 \mathrm{~s} 3 \mathrm{~d}\left({ }^{3} \mathrm{D}\right)$ & $1 \mathrm{~s} 3 \mathrm{~s}\left({ }^{1} \mathrm{~S}_{0}\right)$ & 353.49 & $4 d\left({ }^{2} D\right)$ & $3 \mathrm{~d}\left({ }^{2} \mathrm{D}_{5 / 2}\right)$ & 435.43 & $4 d\left({ }^{2} D\right)$ & $3 \mathrm{~d}\left({ }^{2} \mathrm{D}_{5 / 2}\right)$ & 774.73 \\
\hline $1 \mathrm{~s} 3 \mathrm{~d}\left({ }^{1} \mathrm{D}\right)$ & $1 \mathrm{~s} 3 \mathrm{p}\left({ }^{3} \mathrm{P}_{0}^{\mathrm{o}}\right)$ & 353.52 & $4 f\left({ }^{2} \mathrm{~F}^{\mathrm{o}}\right)$ & & & $4 f\left({ }^{2} \mathrm{~F}^{\mathrm{o}}\right)$ & & \\
\hline $1 \mathrm{~s} 3 \mathrm{p}\left({ }^{1} \mathrm{P}^{\mathrm{o}}\right)$ & $1 \mathrm{~s} 3 \mathrm{p}\left({ }^{3} \mathrm{P}_{1}^{\mathrm{o}}\right)$ & 353.52 & & & & & & \\
\hline & $1 \mathrm{~s} 3 \mathrm{p}\left({ }^{3} \mathrm{P}_{2}^{\mathrm{o}}\right)$ & 353.52 & & & & & & \\
\hline & $1 \mathrm{~s} 3 \mathrm{p}\left({ }^{1} \mathrm{P}_{1}^{\mathrm{o}}\right)$ & 354.51 & & & & & & \\
\hline 11-CC & $13-\mathrm{CC}$ & & $10-\mathrm{CC}$ & 9-CC & & $10-\mathrm{CC}$ & 9-CC & \\
\hline
\end{tabular}

self-consistent. The total recombination cross sections, $\sigma_{\mathrm{RC}}$, for $\mathrm{C}_{\mathrm{IV}}$ and $\mathrm{C} \mathrm{V}$ are obtained from the photoionization cross sections, $\sigma_{\mathrm{PI}}$, and DR collision strengths, $\Omega_{\mathrm{DR}}$, calculated in Nahar and Pradhan (1997) and Pradhan and Zhang (1997). However, the computations for the cross sections are repeated with a much finer energy mesh in order to delineate the detailed resonance structures as observed in the experiments. The eigenfunction expansions for the He-like ions $\mathrm{O}$ VII and $\mathrm{C} v$ include the same configuration interactions (CI). The target terms and fine structure levels included for the three ions are given in table 1. For clarity in comparison with experiment, the target energies are given in $\mathrm{eV}$ in table 1. Energies are specified only for the fine structure components since the $L S$ term energies can be obtained from the statistical average of the fine structure ones. As the CC expansion includes up to the $n=3$ terms, the energy range covered in the calculations is sufficiently large to enable accurate cross sections to be computed over an extended range. However, since the experiments reported cover the range below the $n=2$ terms, we delineate this region to obtain all possible resonances. The theoretical results contain considerably more resonance structures than observed experimentally.

Computations of photoionization cross sections, $\sigma_{\mathrm{PI}}$, in both the non-relativistic ( $L S$ coupling) and the relativistic BP (intermediate-coupling) approximations (Scott and Taylor 1982 ) are carried out using the Opacity Project $R$-matrix codes (Berrington et al 1987) extended for the Iron Project (Hummer et al 1993, Berrington et al 1995) to include the relativistic effects in the BP approximation (see also Zhang 1998). The transition probabilities for the scattering matrix for DR collision strengths are calculated from $f$-values obtained from CC calculations using the same CC expansions or from the atomic structure code SUPERSTRUCTURE (Eissner et al 1974). The DR collision strengths for the $L S$ coupling and the BP cases are obtained using extensions of the $R$-matrix asymptotic region codes, STGFDR (NP1) and RSTGFDR (Zhang and Pradhan 1997), respectively.

The $R$-matrix calculations are carried out for each total angular momentum symmetry $S L \pi$, or $J \pi$, corresponding to a set of fine structure target terms $S_{t} L_{t}$, or levels $J_{t}$. As we shall see from the results, the background recombination cross sections for the highly charged ions considered in this work are very small, and high partial-wave contributions from $J \pi$ 
symmetries with large total angular momentum $J$ in the BP case, and from $S L \pi$ symmetries with large $L$ in the $L S$ case, do not contribute significantly. Resonances corresponding to high electron angular momenta with $\ell>3$ are very narrow, with small autoionization widths. In most practical calculations of $\mathrm{CC}$ cross sections, with very fine resolution, it is found that such resonances do not contribute significantly to the total rate coefficients. Present results (and comparison with experiments) show that it is not necessary to resolve them individually since they are damped out from the effective cross sections (discussed later).

The partial angular momenta in the $L S$ calculations for $\mathrm{H}$-like targets $\mathrm{C}$ VI and $\mathrm{O}$ VIII are $\ell \leqslant 10$ and $L \leqslant 10$, and for He-like target $\mathrm{C} \vee \ell \leqslant 9$ and $L \leqslant 10$. In the BP calculations for $\mathrm{e}^{-}+\mathrm{Cv}_{\mathrm{V}} \rightarrow \mathrm{C}_{\text {Iv }}$, the target involves 13 fine-structure levels (table 1). The total $\left(\mathrm{e}^{-}+\right.$ion) symmetries included are $J=\frac{1}{2}-\frac{9}{2}$ for even parity, and $J=\frac{1}{2}-\frac{11}{2}$ for the odd parity. To account for the relativistic intermediate coupling both the doublets and the quartets (total $\mathrm{e}^{-}+$ion spin $S=\frac{1}{2}$ and $\frac{3}{2}$, respectively) must be included, whereas in $L S$ coupling only the doublets are needed to couple to the target ground state. For $\mathrm{e}^{-}+\mathrm{C}$ VI $\rightarrow \mathrm{Cv}$ and $\mathrm{e}^{-}+\mathrm{O}$ VIII $\rightarrow \mathrm{O}$ VII the total $\left(\mathrm{e}^{-}+\right.$ion) symmetries included are $J=0-4$ for the even and $J=0-5$ for the odd parity. For this case, both the singlet and the triplet $S L \pi$ 's are coupled together in either the $L S$ or the BP intermediate-coupling calculations.

In general, the calculations are divided into two energy ranges depending on whether the background contribution is negligible or not. The criterion refers not simply to the magnitude of the cross sections but also to the overall contribution to the rate coefficients. In the first region, at lower energies from threshold up to about $n=n_{\max }=10$ ( $n$ being the principal quantum number of the outer orbital of the recombined ion bound state), detailed photorecombination cross sections are calculated as in equation (5). In the second region, $n_{\max }<n<\infty$ and $-z^{2} / n_{\max }^{2}$ below each threshold target level, where the resonances are narrow and dense and the background is negligible, we compute the detailed and the resonance-averaged DR cross sections. Here we take $n_{\max }=10$ for all cases. It is necessary to use extremely fine energy meshes in order to delineate the resonance structures belonging to each $n$-complex. The electrons in the first energy range recombine to a large number of final $\left(\mathrm{e}^{-}+\right.$ion) states and recombination cross sections are computed for all coupled symmetries and summed to obtain the total $\sigma_{\mathrm{RC}}$. The number of these final recombined states in the BP case is larger, owing to more channels involving fine structure, than the $L S$-coupling case.

Another relativistic method was used to obtain the DR structures for the purpose of comparison. The fully relativistic distorted-wave (RDW) method described in Sampson and Zhang (1995) is used in which all configuration mixing is included among states, $\left[\left(J_{t}^{\prime \prime}\right) n \ell j\right]_{J}$, in a complex, that is having the same set of $n$ values, parity and total angular momentum $J$. For example, for the $n=5$ complex in the $\mathrm{O}$ VII case, the mixing is included for configurations $2 \mathrm{~s} 5 \mathrm{~s}, 2 \mathrm{~s} 5 \mathrm{~d}, 2 \mathrm{~s} 5 \mathrm{~g}, 2 \mathrm{p} 5 \mathrm{p}$ and $2 \mathrm{p} 5 \mathrm{f}$ for the even parity, and $2 \mathrm{~s} 5 \mathrm{p}, 2 \mathrm{~s} 5 \mathrm{f}, 2 \mathrm{p} 5 \mathrm{~s}, 2 \mathrm{p} 5 \mathrm{~d}$ and $2 \mathrm{p} 5 \mathrm{~g}$ for the odd parity states. For the $n>5$ complex the bound electron orbitals are included only up to $\ell=4$. In this method, the calculations are done for the autoionization and radiative decay rates for the individual autoionizing levels belonging to successive $n$-complexes up to $n \leqslant 15$ only. The energy-dependent cross sections are thereupon derived by the 'sharp resonance' approximation as given in Hahn (1985).

The experimental resolution, albeit very high, represents an 'average' over the electron beam width, and the theoretical results are suitably convolved over a Gaussian distribution corresponding to the measured widths. The computed resonances are resolved on a fine mesh to enable the fitting procedure for radiation damping described in the previous section. We note that the experimental beam distributions are themselves rather complicated, with energy variations and with transverse and parallel components (e.g. Wolf et al 1991), and slight deviations might therefore affect the peak values of some resonances in the convolved cross 
sections. However, as the comparison between theory and experiment shows, this does not appear to pose a significant problem.

\section{Results and discussion}

The primary aim of the present work is to ascertain the accuracy of the CC method for electronion recombination cross sections, $\sigma_{\mathrm{RC}}$, by detailed comparisons with available experimental data. We discuss three sets of calculations for each ion: (a) $L S$ coupling, (b) BP $R$-matrix with no radiative damping, $\mathrm{BP}(\mathrm{NRD})$ and (c) with radiative damping, $\mathrm{BP}(\mathrm{RD})$. For O VIII the RDW results are also reported. Comparison of (a) and (b) demonstrates the effect of relativistic effects and fine structure; (b) and (c) show the extent of radiation damping. The final theoretical results including radiation damping and fine structure, $\mathrm{BP}(\mathrm{RD})$, are compared with the experiment.

\section{1. $e^{-}+C_{V} \rightarrow C$ IV}

Theoretically, the measured DR structures correspond to photorecombination cross sections with resonances

$$
\mathrm{e}^{-}+1 \mathrm{~s}^{2}{ }^{1} \mathrm{~S}_{0} \rightarrow\left(1 \mathrm{~s} 2 \ell^{3,1} L_{J}\right) n \ell^{\prime} \rightarrow\left(1 \mathrm{~s}^{2}{ }^{1} \mathrm{~S}_{0}\right) n \ell^{\prime}+h v .
$$

As the present calculations include the background and the resonances in an ab initio manner, the theoretical cross sections can be compared directly with the absolute experimental cross sections.

In figure 1 the total (a) $L S,(b) \mathrm{BP}(\mathrm{NRD})$ and $(c) \mathrm{BP}(\mathrm{RD})$ recombination cross sections $\sigma_{\mathrm{RC}}$ are shown, with finely delineated resonance structures in both the energy ranges $(n \leqslant 10$ and $10<n \leqslant \infty)$ up to the series limits corresponding to the 1 s $2 \ell$ thresholds; in the former energy range the cross sections are computed at over 3000 energies in the $L S$ case and 24600 energies in the BP case. To make a direct comparison with the experiment (Kilgus et al 1990), we use the reported $2.1 \mathrm{eV}$ FWHM Gaussian distribution to convolve the cross sections and show the results in figure 2 . It can be seen from figure 1 that $(a)$ the $L S$ and $(b)$ the BP(NRD) results are almost identical (except that the resonances are better resolved due to a much finer energy mesh in the BP case), indicating that (a) the relativistic effects are negligible, and (b) the $L S$ and the BP formulations and computations are self-consistent and accurate. From the point of view of numerical accuracy of the present methods it is rather significant that although the BP calculations involve considerably more recombination channels than the $L S$ calculations, owing to nearly twice as many fine structure levels as $L S$ terms, the final results do not show a significant difference between the two.

The two sets of convolved BP results, with and without radiation damping, are shown in figure 2(a) - full and broken curves, respectively-showing the radiative reduction in autoionization. The $\mathrm{BP}(\mathrm{RD})$ results agree very well with the experiment (figure 1 in Kilgus et al (1990) is reproduced here as figure 2(b)). Some discrepancy between the theoretical and experimental results could be expected close to the several series limits shown in the experimental plot, figure $2(b)$. At the $2{ }^{3} \mathrm{P}$ threshold the ' $n$ ' value of the resonances $2{ }^{1} \mathrm{P} n \ell$ is approximately $n=34$. Whereas the resonances in this region, $n>34$, have an additional mode of autoionization into the $2{ }^{3} \mathrm{P}$ continuum that is included in the coupledchannel calculations, the theoretical calculations include all contributions up to $n=\infty$. Experimentally, however, the field ionization of these very high- $n(34<n \leqslant \infty)$ resonance complexes, and certainly of the high orbital angular momentum sublevels $(\ell \geqslant 4$ according to Wolf et al 1991), might take place. The good agreement between theory and experiment 


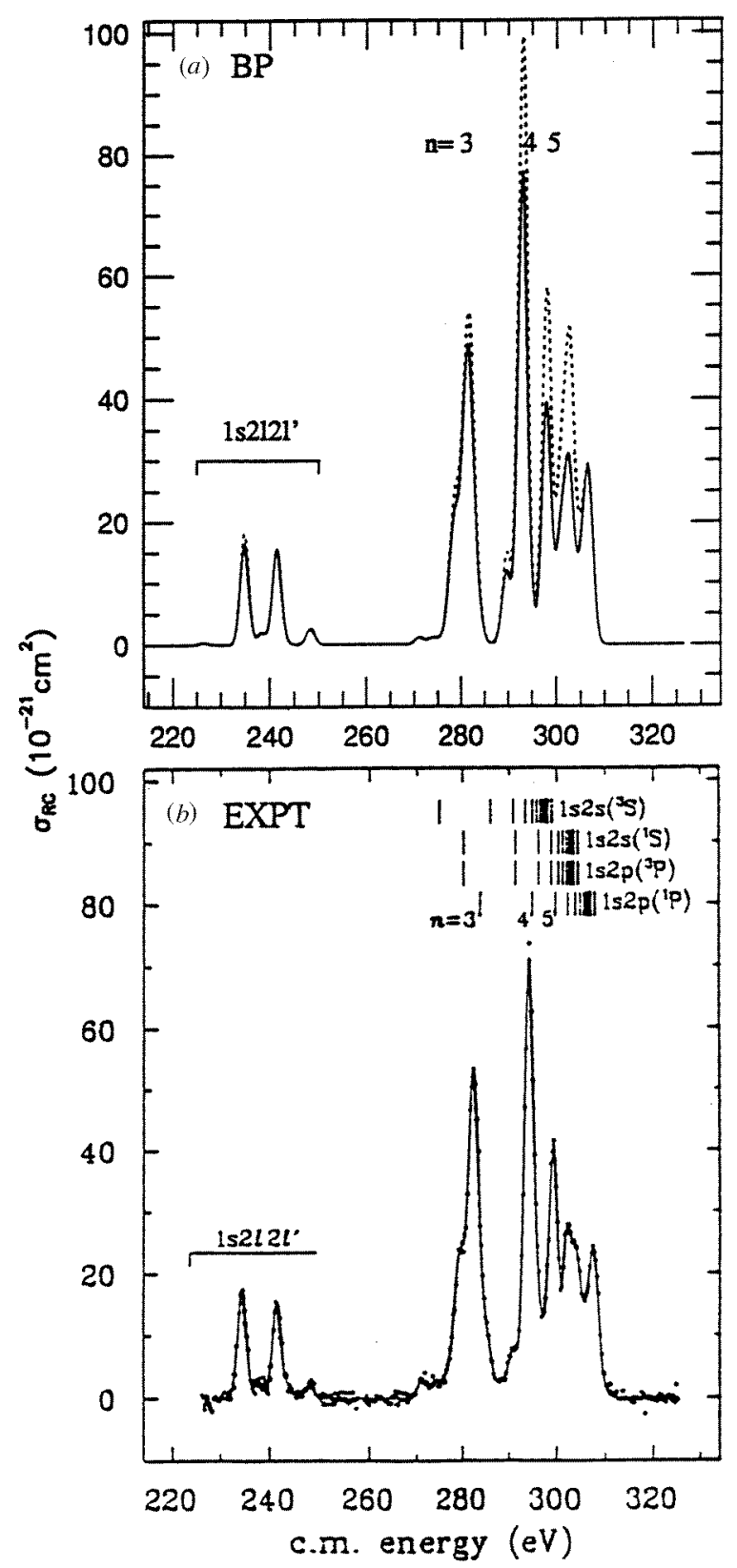

Figure 2. Comparison of (a) the calculated recombination cross sections in the BP approximation including the radiation damping effect with $(b)$ the experimentally measured values (Kilgus et al 1993) for $\mathrm{e}^{-}+\mathrm{Cv} \rightarrow \mathrm{C}$ iv. The theoretical cross sections have been convolved over a $2.1 \mathrm{eV}$ Gaussian beam distribution.

implies that the field ionization of high- $n$ states appears to have a small effect on the effective cross section. It might also be noted that the experimental results show mainly the resonance structures, with the background nearly zero, but the theoretical results in figure 1 also show the background recombination cross sections (on a log scale), which though small could be a 
factor in the calculation of total $\left(\mathrm{e}^{-}+\right.$ion $)$recombination rates averaging over the entire energy range.

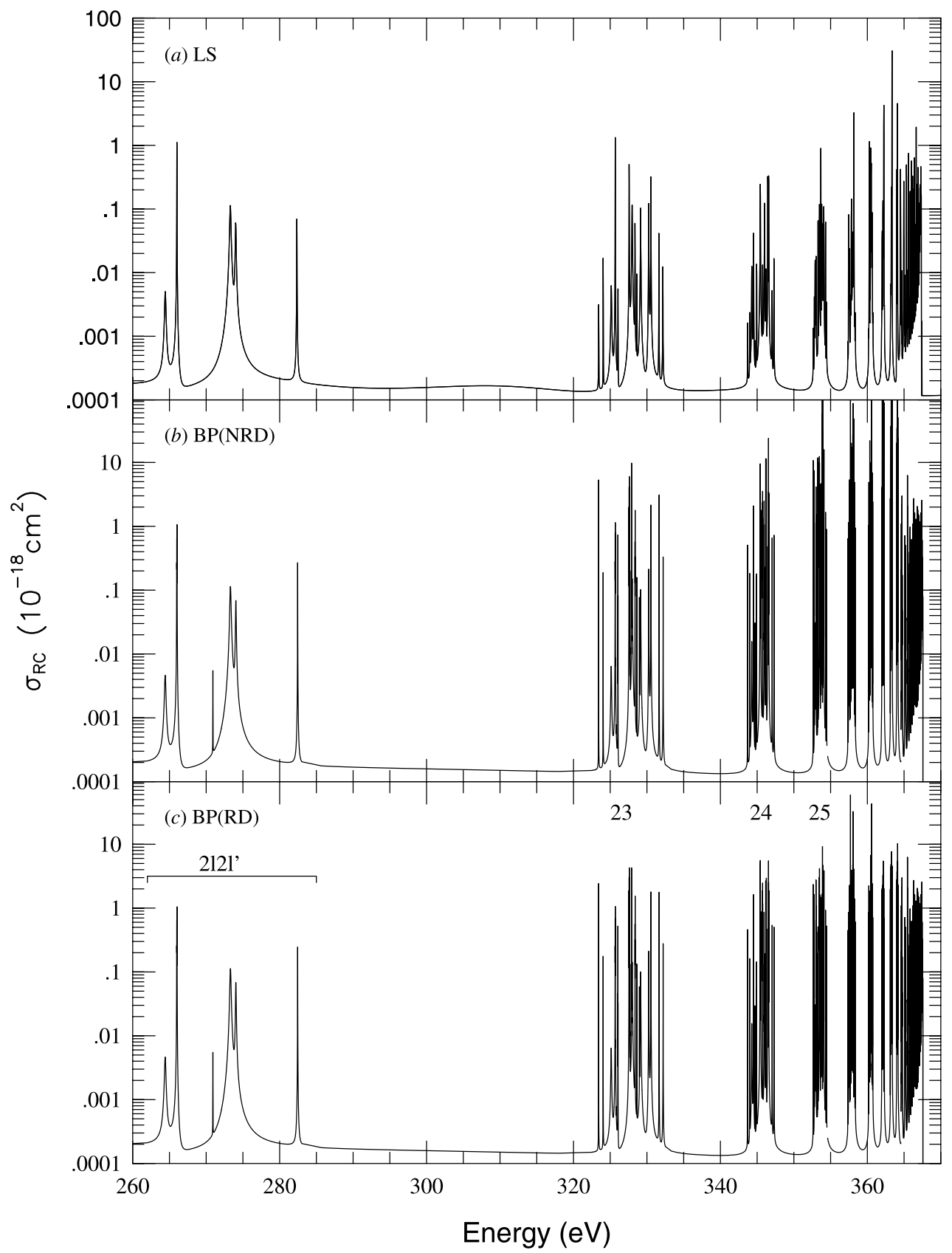

Figure 3. Detailed recombination cross sections, $\sigma_{\mathrm{RC}}$, for $\mathrm{e}^{-}+\mathrm{C} \mathrm{VI} \rightarrow \mathrm{Cv}$ : $(a)$ in the nonrelativistic $L S$-coupling approximation, and in the relativistic Breit-Pauli approximation $(b)$ without radiation damping, $\mathrm{BP}(\mathrm{NRD})$ and $(c)$ with radiation damping, $\mathrm{BP}(\mathrm{RD})$. 


\section{2. $e^{-}+C V I \rightarrow C V$}

The $\mathrm{C}$ vi target (table 1) includes up to $n=4$ terms in the $L S$ calculations and up to $n=3$ fine structure levels in the BP calculations. The experimental cross sections (Wolf et al 1991) are in the range below $400 \mathrm{eV}$, i.e. somewhere above the $n=2$ levels, but well below $n=3$. We therefore confine the comparison with experiment to the region up to about the $n=2$ levels. Figure 3 shows the detailed photorecombination cross sections: $(a)$ the $L S$ and $(b)$ $\mathrm{BP}(\mathrm{NRD})$ and $(c) \mathrm{BP}(\mathrm{RD})$. Resonance groups associated with the $2 \mathrm{p} n \ell$ configurations, $n \geqslant 2$, are clearly separated for the first few $n$-complexes, but each is comprised of all resonances $\ell \leqslant n-1$. These cross sections are convolved with the Gaussian distribution of $2.1 \mathrm{eV}$. The cross sections are computed at over 4000 energies for the $L S$ calculation and over 17000 energies for the BP calculation. Figure 4 compares the convolved $\mathrm{BP}(\mathrm{RD})$ results with the experimental measurements. In the experiment, the critical value of $n_{0}$ equals 11 , above which the partial-wave contributions with $\ell \approx 4$ and higher are estimated to be susceptible to field ionization (Wolf et al 1991).

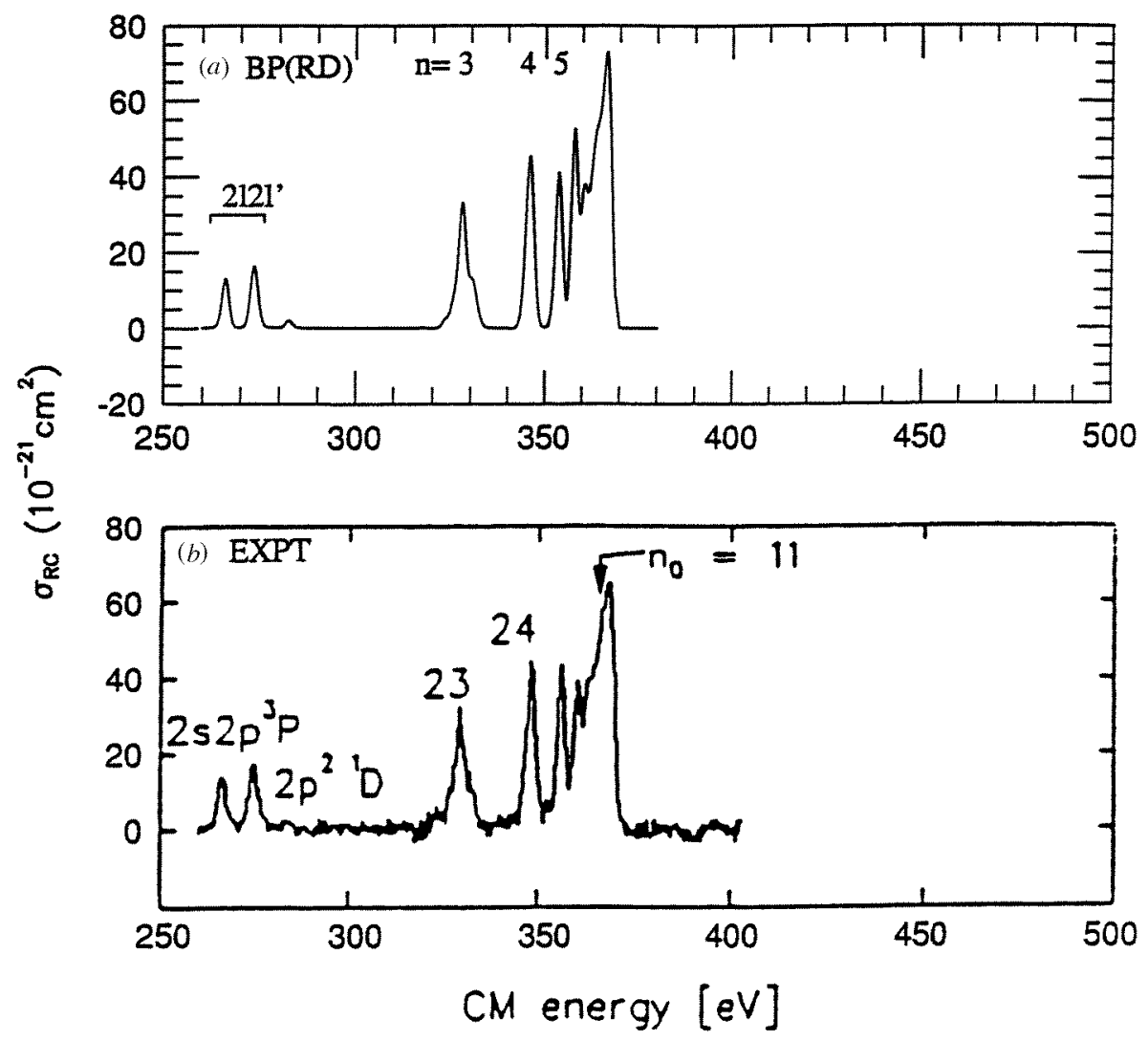

Figure 4. Comparison of $(a)$ the calculated recombination cross sections for $\mathrm{e}^{-}+\mathrm{C} \mathrm{vI} \rightarrow \mathrm{C}$ v in the $\mathrm{BP}$ approximation including the radiation damping effect, with $(b)$ the experimentally measured cross sections (Wolf et al 1991). The theoretical cross sections have been convolved over a $2.1 \mathrm{eV}$ Gaussian distribution corresponding to reported beam widths. 
The theoretical results compare very well with the experimental data and we note the following:

(a) the theoretical cross sections do not exclude any $n$ or $\ell$ contributions and the reported results include all resonance complexes up to $n=\infty$;

(b) the experimental and theoretical cross sections show clearly resolved structures and agree well with each other both in magnitude and shape for all resonance groups (including some very tiny features such as the slight bump on the high-energy side of the '23', i.e. $2 \mathrm{p} 3 \ell$, feature-figure $4(b))$ and

(c) the contribution of field-ionized high- $n$ or large- $\ell$ states in the experiment appears to be rather small.

4.3. $e^{-}+O$ VIII $\rightarrow O$ VII

The experimental report (Kilgus et al 1990) for O vIII recombination spectra contains results not only below the $n=2$ thresholds, but also $n=3$. We therefore extend the theoretical calculations up to the $n=3$ levels. Figure 5 shows the detailed CC cross sections for (a) the $L S$, (b) the $\mathrm{BP}(\mathrm{NRD})$ and (c) the $\mathrm{BP}(\mathrm{RD})$ cases. In addition to $L S$ and $\mathrm{BP}$ calculations, we have also carried out relativistic distorted-wave (RDW) calculations for comparison. Figure 6 presents the $\mathrm{BP}(\mathrm{RD})$ cross sections convolved over a $2.1 \mathrm{eV}$ Gaussian, together with results from cross sections derived from the RDW rates, and the experimental data from (Kilgus et al 1990). The $L S$ and the BP(NRD) results for O viII, figures 5(a) and (b), differ more than for the carbon ions owing to the relativistic effects and additional fine structure channels in the latter case. The radiatively damped BP results in figure $6(b)$ agree well with the RDW results in figure 6(a), and the experimental results in figure 6(c).

It is noted that the recombination cross sections above the $n=2$ levels are considerably smaller than those below, due to autoionization of the $3 \ln \ell^{\prime}$ resonances into the excited $2 \ell$ levels. Autoionization into excited states, and consequent reduction in DR, is an important effect in electron-ion recombination as it determines the relative contribution to the total recombination rate from resonances converging on to excited target thresholds. It is also responsible for the differences in the new total recombination rates computed using the $\mathrm{CC}$ method (NP1, NP2, Nahar and Pradhan 1997) from the Burgess general formula (1965) that does not include autoionization into excited states. For complicated systems, such as iron ions Fe I, II and III, with many excited target states (between 50 to 100), the reduction in the recombination rate coefficients at high temperatures can be substantial. As the $\mathrm{O}$ viII results in figure 6 show, the contribution of resonances above the $n=2$ levels to the recombination rate is likely to be negligibly small.

\subsection{Correspondence between photorecombination, dielectronic recombination and excitation}

The present close-coupling treatment of (electron-ion) recombination is a unified and integrated approach to photorecombination (PR), DR and electron impact excitation (EIE). For example, as required by conservation of flux, the independently computed total cross sections for PR, DR and EIE for $\mathrm{e}^{-}+\mathrm{C}$ VI $\rightarrow \mathrm{Cv}$ are a continuous function of energy, as illustrated in figure 7. The PR cross sections include the background non-resonant contribution as well as the resonances (left of the broken line in figure 7), whereas the DR cross sections (right of the broken line), computed using the BS theory, neglect the background contribution. The two cross sections, the PR and DR, match smoothly at $v \approx 10.0$ showing that the background contribution is negligible compared to the resonant contribution at high $n>10$. Further, 


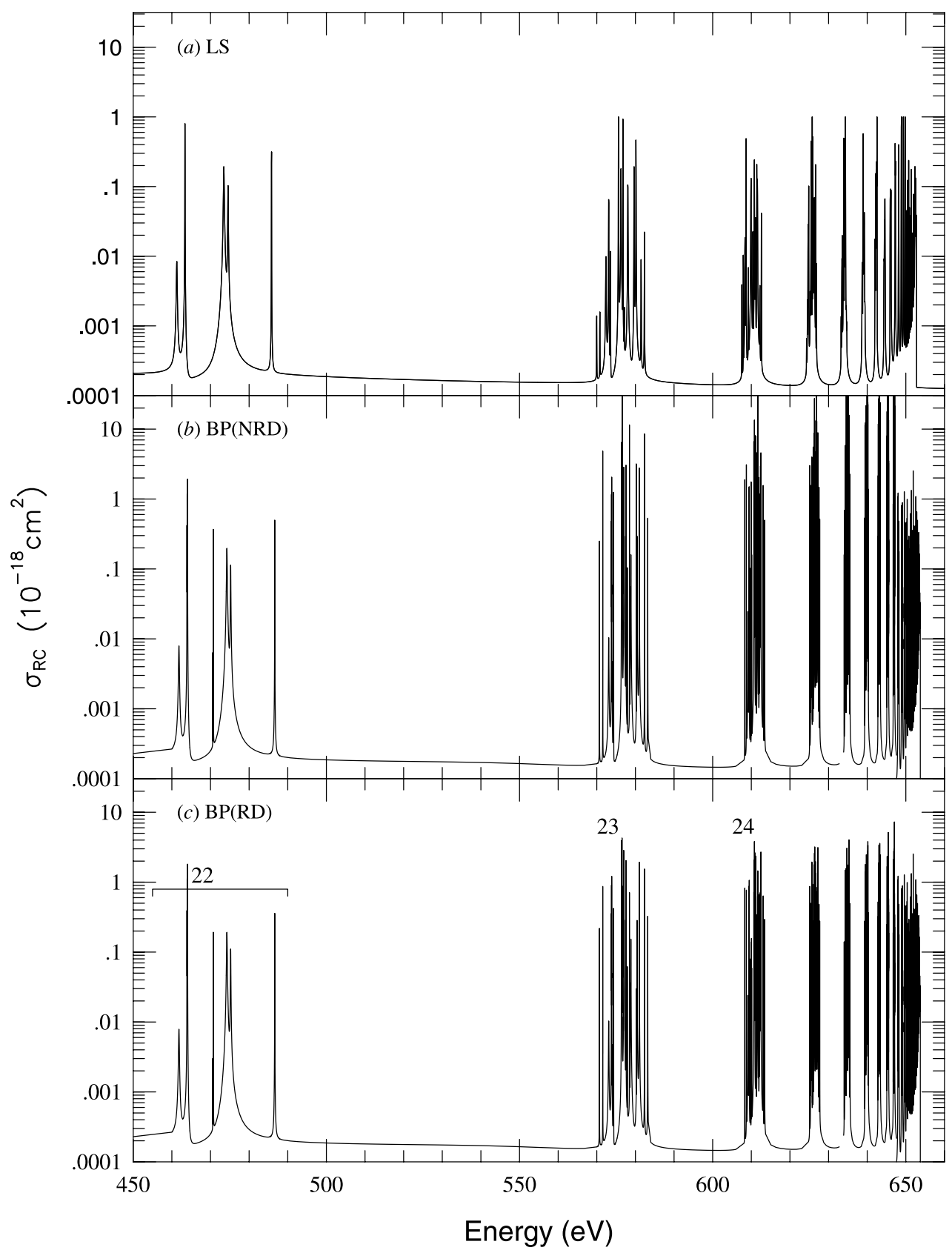

Figure 5. Detailed recombination cross sections, $\sigma_{\mathrm{RC}}$, for $\mathrm{e}^{-}+\mathrm{O}$ VIII $\rightarrow \mathrm{O}$ VII: $(a)$ in the $L S$ coupling approximation, and in the relativistic Breit-Pauli approximation $(b)$ without radiation damping, $\mathrm{BP}(\mathrm{NRD})$ and $(c)$ with radiation damping, $\mathrm{BP}(\mathrm{RD})$.

the DR cross sections rise exactly up to the EIE cross section at the threshold of excitation according to the theoretical condition

$$
\lim _{n \rightarrow \infty} \Omega_{\mathrm{DR}}(n)=\lim _{k^{2} \rightarrow 0} \Omega_{\mathrm{EIE}}\left(k^{2}\right) .
$$



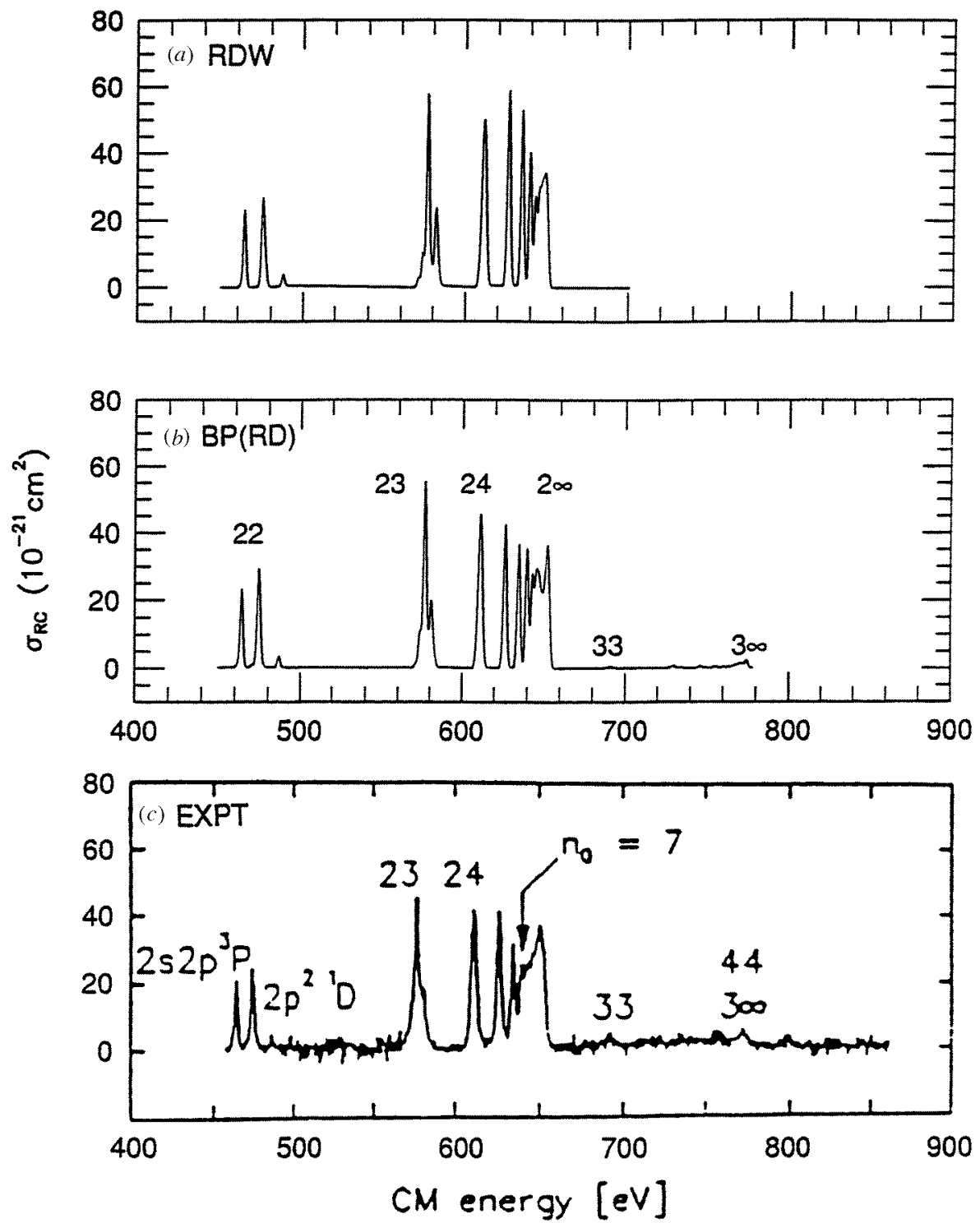

Figure 6. Comparison of the calculated recombination cross sections for $\mathrm{e}^{-}+\mathrm{O}_{\mathrm{VIII}} \rightarrow \mathrm{O}$ VII in (a) the relativistic distorted-wave (RDW) approximation, and $(b)$ the BP approximation including the radiation damping effect, with $(c)$ the experimentally measured cross sections (Wolf et al 1991). The theoretical cross sections have been convolved over a $2.1 \mathrm{eV}$ Gaussian distribution corresponding to the reported beam widths.

The DR cross section in figure 7 at the series limit $2{ }^{1} \mathrm{P}_{1}$ agrees precisely with the independently determined value of the electron impact excitation cross section (full circle) for the dipole transition $1{ }^{1} \mathrm{~S}_{0}-2{ }^{1} \mathrm{P}_{1}$, as required by the unitarity condition for the generalized $S$-matrix and conservation of flux. The continuous transition between the PR, DR and EIE cross sections serves to validate the accuracy of the BS theory of DR. The DR cross sections are, on the one hand, consistent with an extensively detailed coupled-channel treatment of 


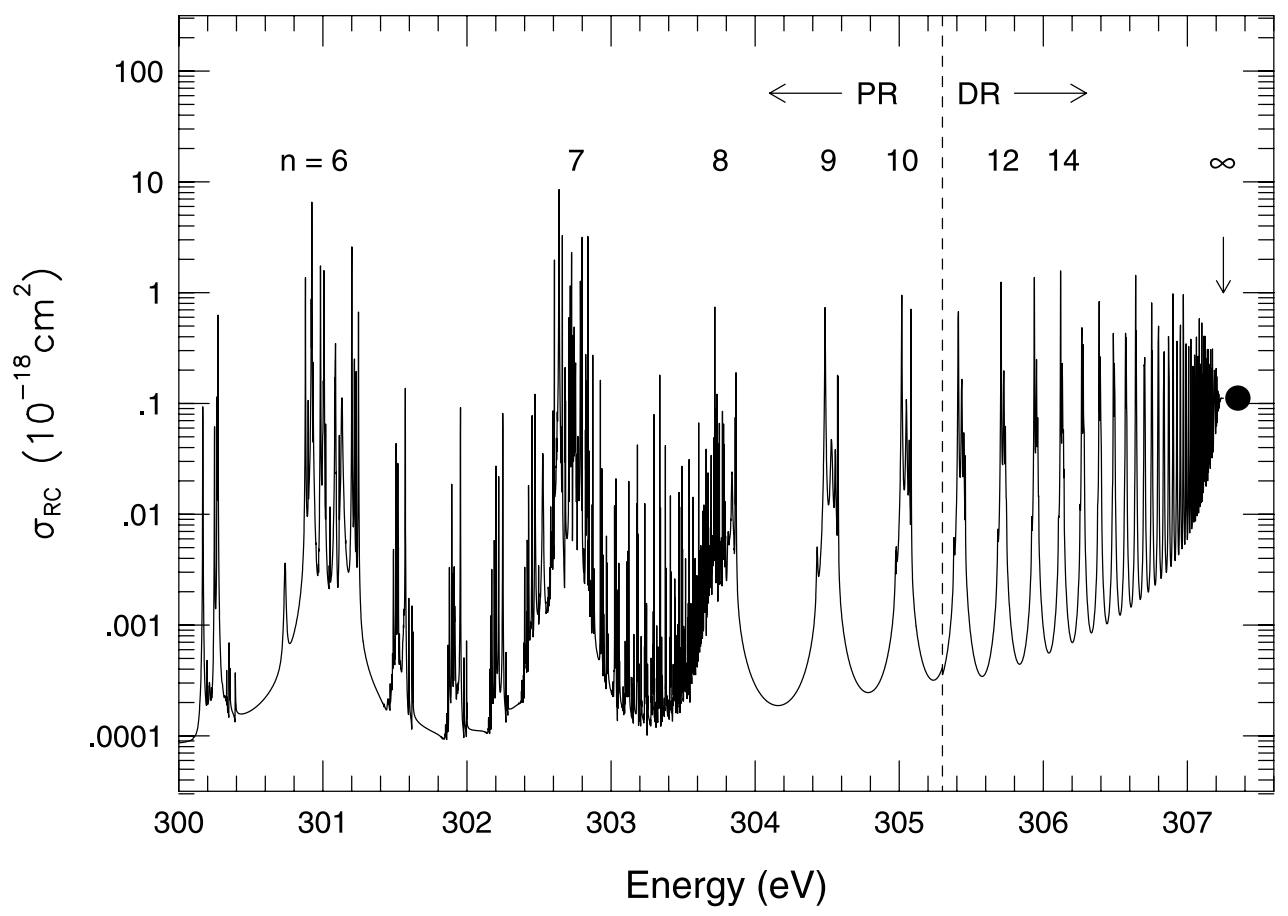

Figure 7. Photo-recombination (PR) cross sections, as derived from photoionization calculations (left of the broken line) and the dielectronic (DR) cross sections obtained using the Bell and Seaton theory (right of the broken line) for $\mathrm{e}^{-}+\mathrm{Cv} \rightarrow \mathrm{C}$ Iv; the full circle represents the near-threshold value of electron impact excitation cross section for the dipole transition $1{ }^{1} \mathrm{~S}_{0}-2{ }^{1} \mathrm{P}_{1}^{0}$ in $\mathrm{C}$ v.

photorecombination, until an energy region where background recombination is insignificant, and, on the other hand, consistent with the threshold behaviour at the EIE threshold. In fact, equation (17) provides a powerful accuracy check on the possible importance of longrange multipole potentials, partial-wave summation, level degeneracies at threshold and other numerical inaccuracies (discussed in previous works).

In a recent work Robicheaux $\dagger$ (1998) has discussed the approximations made in the BS theory of DR in the high- $n$ limit in a very small region just below the EIE threshold. In this region the $n$ are very large ( $>100)$ and the Rydberg spacing is smaller than the radiative decay rate of the core. Seaton (1998) has further elaborated on this region in detail, showing that the ratio of DR rates from the BS theory and the formulation by Robicheaux are of the order of $10^{-7}$ for all values of energy. As such, there should not be any discernible effect on the DR cross sections and rate coefficients.

The radiatively damped cross sections in figure 7 illustrate that, owing to the interaction with the radiation field, the autoionizing resonances are broadened, smeared and wiped out (in that order) as $n \rightarrow \infty$. At sufficiently high $n$ the resonant contribution (DR) is very large compared to the background, non-resonant photorecombination (PR) cross section. In the unified method of electron-ion recombination, for $n>n_{\max }$, we employ the BS theory to compute the detailed and the averaged resonant DR cross sections; the background

$\dagger$ Pradhan and Zhang (1997) ascribed to Robicheaux et al (1995) the assertion that the BS theory leads to 'strange poles in the $S$-matrix'. However, the quoted phrase was taken from a preprint of the Robicheaux et al paper, and did not appear in the final, published version. The error is regretted. 
contributions are computed in the hydrogenic approximation. The agreement and the continuity between the three sets of data in figure 7 shows the $\mathrm{CC}$ method for electron-ion recombination to be accurate and self-consistent with inverse photoionization (photorecombination) and electron impact excitation.

\subsection{Relativistic distorted-wave calculations}

The distorted-wave method, in the isolated resonance approximation has been widely employed to obtain DR cross sections (Hahn 1985). In the RDW calculation the autoionization and radiative decay rates are computed individually for each resonance complex and then energydependent cross sections are obtained with a width corresponding to the resonance. The cross sections thus derived are further convolved over a Gaussian distribution to compare with the experimental results. For O viI we also present the DR cross sections computed in this manner using the RDW method (Sampson and Zhang 1995) in figure 6(a). The RDW results are expected to be of reasonable accuracy for highly charged ions, as is found to be the case for recombination with $\mathrm{H}$-like $\mathrm{O}$ viII. However, it should be noted that the background cross section is not included here (it can be added separately from the non-resonant RDW PI cross sections via detailed balance), and the uncertainties in resonance widths and positions may be large for complex atomic systems with many coupled channels with overlapping resonances. On the other hand, the present RDW method accounts fully for the relativistic effects in pure $j j$ coupling in a Dirac-Fock-Slater approximation, and as such is very useful for highly ionized and few-electron systems with high $Z(Z>30)$, where intermediate coupling (i.e. BP) may be increasingly inaccurate.

\subsection{Radiation damping of resonances}

Scattering, photoionization and recombination of all atomic systems involve an infinite number of resonances. Close-coupling calculations allow for resonances in an ab initio manner. Broad low-lying near-threshold resonances have autoionization rates $\Gamma_{\mathrm{a}} \sim 10^{14} \mathrm{~s}^{-1}$, much greater than typical dipole radiative decay rates $\Gamma_{\mathrm{r}} \sim 10^{6-12} \mathrm{~s}^{-1}$. For most atomic systems up to the iron-peak elements $(Z=30), \Gamma_{\mathrm{r}}<10^{12} \mathrm{~s}^{-1}$ for the lowest dipole transitions. Therefore, a priori, radiation damping is not expected to be a dominant effect. Notable exceptions are high- $Z, \mathrm{H}$ - and He-like ions with $\Gamma_{\mathrm{r}} \sim \Gamma_{\mathrm{a}}$. For highly excited states with $n \rightarrow \infty$, the $\Gamma_{\mathrm{a}}$ decreases as $n^{-3}$ and $\Gamma_{\mathrm{r}}$ dominates.

However, all resonances are susceptible to radiation damping somewhere between $0 \%$ and $100 \%$, i.e. damping factors (undamped/damped) from 1 to $\infty$, depending on $n, \ell$, energy relative to threshold, and radiative transition probability $\Gamma_{\mathrm{r}}$ (radiation damping of resonance profiles was first demonstrated in Pradhan (1981), and Pradhan and Seaton (1985)). Determination of damping factors of individual resonances may be inaccurate as it depends on the precise positions, heights and shapes of resonances. In a recent work, Badnell et al (1998) report undamped/damped ratios $>$ unity for a few individual resonance profiles in several atomic systems, concluding that radiation damping is generally important. Their study is incomplete, however, since they do not investigate the effect on all resonances in an ion, or the overall effect on the cross sections throughout the energy range of interest, as done, for example, in the present or our previous works.

The precise positions, shapes and heights of resonances depend not only on resolution, but also on the $\mathrm{CC}$ wavefunction expansions (and accuracy thereof), the various parameters chosen for $R$-matrix calculations, and numerical inaccuracies. Resolution per se is not a major problem by comparison. All radiative decay rates in neutrals, near-neutrals and heavy atomic systems in 
general are negligibly smaller than the autoionization widths of the near-threshold resonances. Badnell et al do not present any complete or detailed CC calculations except for He-like C v, and a model calculation for Ne-like Fe XVII in $L S$ coupling (neglecting the considerable fine structure known to be present (Smith et al 1985)). Their results for all other ions are obtained using perturbative methods, expected to be accurate for few-electron, highly charged ions but may not give precise undamped/damped ratios for extremely narrow resonances. Although Badnell et al quote these factors to high precision (such as '1.06' for $n=4$ and ' 1.73 ' for $n=8$ in Al-like Fe xIV), these damping factors cannot be so accurate. They depend not only on ' $n$ ', but on all the quantum numbers $\left(S_{i} L_{i} J_{i}\right) n \ell[S L J]$ of each resonance in a given $n$ manifold. Our fully relativistic RDW calculations in pure $j j$-coupling (a perturbative method with high accuracy) indicate such factors to be much more uncertain, even discounting the effect of channel couplings, uncertainties in target and correlation wavefunctions, resonance overlaps, etc.

More meaningful than the damping factors for individual resonances is the combined effect of all damped resonances on the integrated rate coefficient for an atomic process, i.e. on the quantities of practical interest in laboratory and astrophysical applications. Rate coefficients are usually obtained by averaging cross sections over a Maxwellian. Generally, broad nearthreshold resonances corresponding to the lowest $n$ and $\ell$ make the dominant contribution, relative to narrower ones with higher $n$ and $\ell$. The CC calculations include all closed channels with $n \leqslant 10$ and $\ell \leqslant n-1$. Other methods based on quantum defect theory, such as Gailitis averaging of resonances, or the BS theory of DR, are employed for $10<n \leqslant \infty$. It appears that the pre-convolution method of Badnell et al (1998), based on MCQDT, would be suitable for detailed studies of resonances that are neglected. However, the MCQDT analysis may not be very accurate for low- $n(n<10)$ resonances, including relativistic fine structure, in complex systems that are of main interest.

As mentioned, radiative decays are significant for highly charged $\mathrm{H}$ - and He-like ions. Table 2 shows the dielectronic satellite recombination rates for $\mathrm{e}^{-}+\mathrm{Fe} \mathrm{xxv} \rightarrow \mathrm{Fe}$ xxIv obtained using the unified treatment of recombination in the Breit-Pauli $R$-matrix approximation (BPRM), as well as the relativistic distorted-wave (RDW) approximation, and compared to similar calculations by Badnell et al (1998) $\dagger$. While the undamped BPRM cross sections expectedly differ from the RDW results, owing to the neglect of very narrow high- $\ell$ resonances, the final results allowing for radiative damping of included resonances are nearly exactly the same. Any additional contribution to the undamped cross sections due to unresolved resonances must be damped out since higher resolution does not yield different results. It has further been verified, through extensive and fully relativistic RDW calculations in $j j$-coupling for a complex of 130 resonances belonging to the $1 \mathrm{~s} 2 \ell 6 \ell^{\prime}$ complex (Zhang, unpublished), that the neglected resonances do not make any partial contributions to the total rate.

The primary application of the present $\mathrm{CC}$ method for $\left(\mathrm{e}^{-}+\mathrm{ion}\right)$ recombination is to obtain unified, total recombination rates for elements up to the iron-peak elements with $Z \leqslant 30$ (well within the validity of the intermediate-coupling approximation using the BP method). In general, the contribution from the near-threshold region is dominated by large resonances with $\Gamma_{\mathrm{a}} \sim 10^{14} \mathrm{~s}^{-1}$; the extremely narrow resonances, with $\Gamma_{\mathrm{a}}$ several orders of magnitude smaller, make relatively little contribution and may be neglected. The calculations generally resolve each resonance $n$-complex up to $\ell \leqslant 4$; the higher $\ell$ resonances are assumed to be damped out, thereby making allowance for radiation damping, although it is not a large effect on the final

$\dagger$ The present calculations for Fe xxv showed that there is an error of more than an order of magnitude in one of the undamped resonance peak values of $\sim 2000$ given by Gorczyca and Badnell (1997, figure 1(c)); our calculations give 154. However, according to Dr T Gorczyca (private communication) the error is typographical and their value is about 200. 
Table 2. Photorecombination rate coefficients for Fe xxv at $2 \mathrm{keV}\left(10^{-13} \mathrm{~cm}^{3} \mathrm{~s}^{-1}\right)$. Undamped values depend on the resolution; however, all radiatively damped results are in good agreement.

\begin{tabular}{|c|c|c|c|c|c|c|c|c|}
\hline \multirow[b]{2}{*}{ KLn } & \multicolumn{4}{|c|}{ Undamped } & \multicolumn{4}{|c|}{ Damped } \\
\hline & BPRM $^{\mathrm{a}}$ & $\mathrm{BPRM}^{\mathrm{b}}$ & $\mathrm{RDW}^{\mathrm{a}}$ & BPDW $^{b}$ & BPRM $^{\mathrm{a}}$ & $\mathrm{BPRM}^{\mathrm{b}}$ & $\mathrm{RDW}^{\mathrm{a}}$ & $\mathrm{BPDW}^{\mathrm{b}}$ \\
\hline KLL & 12.8 & 18.6 & 18.3 & 18.9 & 2.49 & 2.455 & 2.46 & 2.417 \\
\hline KLM & 27.0 & 30.2 & 30.0 & 30.2 & 1.10 & 1.117 & 1.11 & 1.033 \\
\hline KLN & 18.9 & 40.6 & 37.8 & 40.9 & 0.375 & 0.377 & 0.414 & 0.380 \\
\hline KLO & 21.5 & 57.1 & 47.6 & 57.5 & 0.192 & 0.179 & 0.198 & 0.180 \\
\hline KLP & 29.2 & 78.2 & 45.1 & 78.5 & 0.123 & 0.096 & 0.114 & 0.100 \\
\hline Total $^{c}$ & 205.1 & - & 378.8 & - & 4.531 & - & 4.587 & - \\
\hline
\end{tabular}

a Present.

b Badnell et al (1998).

c Includes higher $n$-complexes (Badnell et al do not present total rates). Present total rates also agree well with several previous works (see table 16 in Hahn and LaGattuta 1988).

results. With the exception of $\mathrm{H}$ - and He-like ions where all resonances undergo significant radiative damping, the cross sections and rate coefficients should not be influenced by radiation damping in the inner region of the $R$-matrix radius where the core radiative transitions do not significantly compete with autoionization. This is certainly true for all $\Delta n=0$ radiative core transitions even in highly charged ions. For example, the theoretical results without radiation damping of low- $n$ resonances in photorecombination cross sections for Ar XIV (Zhang and Pradhan 1997), are in excellent agreement with the absolute cross sections measured from the storage ring CRYRING, both in magnitude and details of the extensive resonance structures and background cross section.

Finally, it might be pointed out that, as yet, no total recombination rate coefficients have been presented in the literature that are discrepant, due to radiation damping, with those calculated using the unified formulation based on the close-coupling approximation. The narrow resonance structures may differ owing to resolution and accuracy of their energies, widths, heights, and shapes. However, all sets of final results (damped) agree (table 2), as the extremely narrow resonances are either fully damped out or adequately treated by the perturbative method employed in this work.

\section{Conclusion}

The close-coupling method is employed to compute $\left(\mathrm{e}^{-}+\mathrm{ion}\right)$ recombination cross sections and is shown to be in very good agreement with available experimental data of high resolution and accuracy. The agreement between the theoretical cross sections and experimental works lends confidence to the recombination rate coefficients computed in several previous works by the authors using the close-coupling method. In particular, in recent works (Nahar and Pradhan 1997, Nahar 1999) we have presented total, unified recombination rate coefficients for all carbon, nitrogen and oxygen ions. Nahar (1999) also discusses a comparison of the BPRM results for $\mathrm{He}$-like $\mathrm{O}$ vII recombination rates including fine structure and radiation damping. As the present work shows, the relativistic effects, even for recombination with $\mathrm{H}$-like $\mathrm{C}$ and $\mathrm{O}$ ions, are small. Radiation damping effects may be included using perturbative methods. Based on the close agreement with experiments, it appears that it would not be unreasonable to expect an absolute uncertainty in the theoretical results of approximately $10 \%$ in the energy range under consideration. Further studies are in progress for other atomic systems with more complex electron correlation and relativistic effects. 
For most atomic systems both the background and resonant recombination (RR and DR) processes are important and need to be considered. For highly charged ions (such as those considered in this work) DR is usually the dominant recombination process. Although generally applicable to all systems, including highly charged ions as in this work, the closecoupling BPRM method is especially suitable for the strong-coupling cases, such as neutrals and near neutrals, where the broad and overlapping resonances dominate the near-threshold region in the electron-ion recombination process, and other methods may not be accurate.

\section{Acknowledgments}

The authors would like to thank Dr Werner Eissner for invaluable computational assistance and Professor M J Seaton and Dr Keith Berrington for discussions. This work was supported partially by the US National Science Foundation (grant no AST-9870089), and by NASA grant NAG5-6908. The computational work was carried out on the Cray Y-MP8/64 at the Ohio Supercomputer Center in Columbus, $\mathrm{OH}$.

\section{References}

Badnell N R, Gorczyca T W and Price A D 1998 J. Phys. B: At. Mol. Opt. Phys. 31 L239

Badnell N R, Pindzola M S and Griffin D C 1990 Phys. Rev. A 412422

Beiersdorfer P, Schneider M B, Bitter M and von Goeler S 1992 Rev. Sci. Instrum. 635029

Bell R H and Seaton M J 1985 J. Phys. B: At. Mol. Phys. 181589

Berrington K A, Burke P G, Butler K, Seaton M J, Storey P J, Taylor K T and Yu Yan 1987 J. Phys. B: At. Mol. Phys. 206379

Berrington K A, Eissner W and Norrington P H 1995 Comput. Phys. Commun. 92290

Burgess A 1965 Astrophys. J. 1411588

Davies P C W and Seaton M J 1969 J. Phys. B: At. Mol. Phys. 2757

DeWitt D R, Lindroth E, Schuch R, Gao H, Quinteros T and Zong W 1995 J. Phys. B: At. Mol. Opt. Phys. 28 L147

DeWitt D R, Schuch R, Zong W, Asp S, Biedermann C, Chen M H and Badnell N R 1996 Phys. Rev. A 532327

Eissner W, Jones M and Nussbaumer H 1974 Comput. Phys. Commun. 8270

Eissner W 1999 Comput. Phys. Commun. in preparation

Gorczyca T W and Badnell N R 1997 Phys. Rev. Lett. 792783

Hahn Y 1985 Adv. At. Mol. Phys. 21123

Hahn Y and LaGattuta K J 1988 Phys. Rep. 166195

Hummer D G, Berrington K A, Eissner W, Pradhan A K, Saraph H E and Tully J A 1993 Astron. Astrophys. 279298

Kilgus G et al M 1990 Phys. Rev. Lett. 64737

Kilgus G, Habs D, Schwalm D, Wolf A, Schuch R and Badnell N R 1993 Phys. Rev. A 474859

Mannervik S, Asp S, Broström L, DeWitt D R, Lidberg L, Schuch R and Chung K T 1997 Phys. Rev. A 551810

Nahar S N 1995 Astrophys. J. Suppl. 101423

-1996a Astrophys. J. Suppl. 106213

-1996b Phys. Rev. A 532417

-1997 Phys. Rev. A 551980

-1999 Astrophys. J. Suppl. 120131

Nahar S N, and Bautista M A 1999 Astrophys. J. Suppl. 120

Nahar S N, Bautista M A and Pradhan A K 1997 Astrophys. J. 497479

-1998 Phys. Rev. A 585593

Nahar S N and Pradhan A K 1994 Phys. Rev. A 491816

-1995 Astrophys. J. 447966

-1997 Astrophys. J. Suppl. 111339

Pindzola M S, Badnell N R and Griffin D 1990 Phys. Rev. A 42282

- 1992 Recombination of Atomic Ions NATO ASI B p 296

Pradhan A K 1981 Phys. Rev. Lett. 4779

Pradhan A K and Seaton M J 1985 J. Phys. B: At. Mol. Phys. 181631

Pradhan A K and Zhang H L 1997 J. Phys. B: At. Mol. Opt. Phys. 30 L571

Robicheaux F 1998 J. Phys. B: At. Mol. Opt. Phys. 31 L109 
Sakimoto K, Terao M and Berrington K A 1990 Phys. Rev. A 42291

Sampson D H and Zhang H L 1995 J. Quant. Spectrosc. Radiat. Transfer 54345

Seaton M J 1987 J. Phys. B: At. Mol. Phys. 206363

1998 J. Phys. B: At. Mol. Opt. Phys. 31 L1017

Scott N S and Taylor K T 1982 Comput. Phys. Commun. 25347

Smith B W, Raymond J C, Mann J B and Cowan R D 1985 Astrophys. J. 298898

Wolf A et al 1991 Z. Phys. D 21569

Zhang H L 1998 Phys. Rev. A 572640

Zhang H L and Pradhan A K 1997 Phys. Rev. A 78195

Zhang H L, Sampson D H and Mohanty A K 1989 Phys. Rev. A 40616 